\title{
Observations of Anomalous Dust
}

\author{
Richard John Davis, " R.D. Davies \\ University of Manchester, Jodrell Bank Observatory \\ Macclesfield, Cheshire SK11 9DL, UK \\ E-mail: rid@jb.man.ac.uk \\ C. Dickinson, K.M. Gorski \\ Caltech \\ Pasadena CA, USA
}

\section{A.J. Banday, T.R. Jaffe}

Max-Planck-Institut

Garching, Germany

\begin{abstract}
In our study of the free-free, dust and synchrotron foreground components in the WMAP data we have chosen a selection of fields which are intended to have minimal cross-contamination from other components. Each of the 3 components has been quantified in terms of a mean value of the emissivity in each of the 5 WMAP bands. We have made studies of the anomalous dust emission on and off the plane. There is clear evidence for a range of the emissivity of up to 2.5
\end{abstract}

\section{Introduction}

WMAP data when combined with ancillary data on free-free, synchrotron and dust allow an improved understanding of the spectrum of emission from each of these components. Here we examine the sky variation at intermediate latitudes using a crosscorrelation technique. In particulare we compare the observed emission in 15 selected sky regions to three "standard" templates (Davies et al. 2006).

\footnotetext{
${ }^{*}$ Speaker
} 


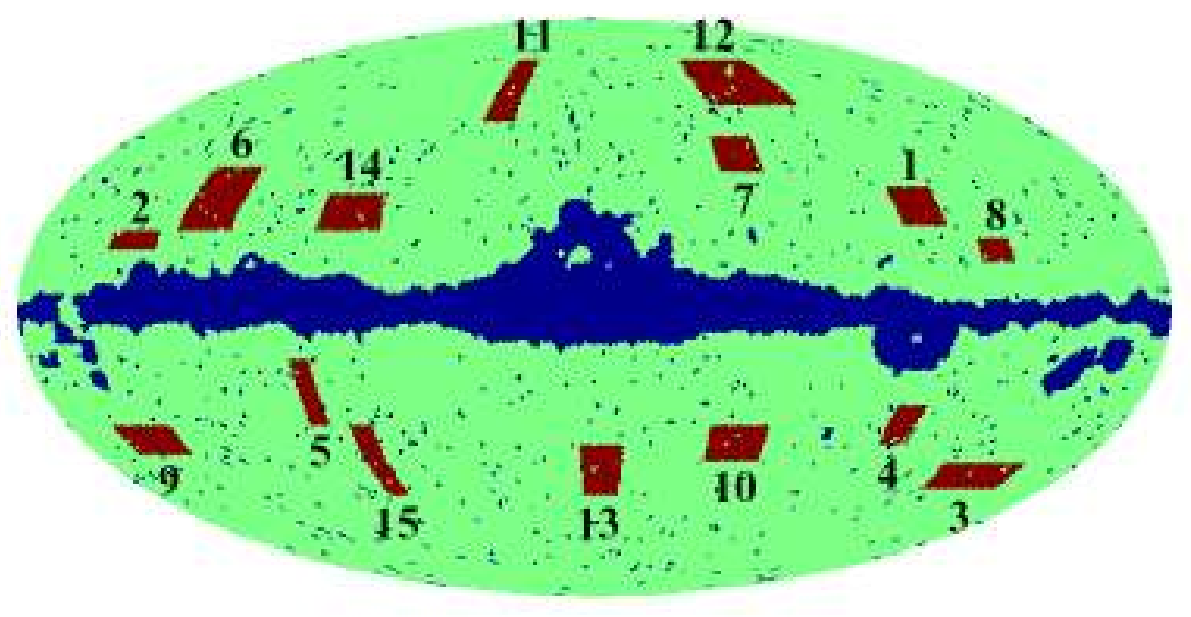

Fig. 1 Full-sky showing 15 selected regions

\begin{tabular}{ccccl}
\hline $\begin{array}{c}\text { Field } \\
\text { No. }\end{array}$ & $\begin{array}{c}\text { Dominant } \\
\text { Emission }\end{array}$ & $\begin{array}{c}\text { Longitude } \\
\text { Range }\end{array}$ & $\begin{array}{c}\text { Latitude } \\
\text { Range }\end{array}$ & Description. \\
\hline 1 & Free-free & $245^{\circ}-260^{\circ}$ & $+21^{\circ}-+31^{\circ}$ & Northern edge of Gum Nebula. \\
2 & Free-free & $140^{\circ}-155^{\circ}$ & $+15^{\circ}-+20^{\circ}$ & Disc-like structure above Galactic plane. \\
3 & Free-free & $200^{\circ}-230^{\circ}$ & $-41^{\circ}--48^{\circ}$ & Eridanus complex - within southern Gould Belt \\
4 & Free-free & $250^{\circ}-260^{\circ}$ & $-25^{\circ}--35^{\circ}$ & Southern edge of Gum Nebula \\
5 & Free-free & $90^{\circ}-97^{\circ}$ & $-13^{\circ}--30^{\circ}$ & Below plane in northern sky. \\
\hline 6 & Dust & $118^{\circ}-135^{\circ}$ & $+20^{\circ}-+37^{\circ}$ & l=125 dust spur, NCP region (the "duck"). \\
7 & Dust & $300^{\circ}-315^{\circ}$ & $+35^{\circ}-+45^{\circ}$ & Outer edge of northern Gould Belt system. \\
8 & Dust & $227^{\circ}-237^{\circ}$ & $+12^{\circ}-+18^{\circ}$ & Above plane in southern sky. \\
9 & Dust & $145^{\circ}-165^{\circ}$ & $-30^{\circ}--38^{\circ}$ & Orion region in southern Gould Belt. \\
10 & Dust & $300^{\circ}-320^{\circ}$ & $-30^{\circ}--40^{\circ}$ & Below plane southern sky. \\
\hline 11 & Synchrotron & $33^{\circ}-45^{\circ}$ & $+50^{\circ}-+70^{\circ}$ & Middle section of North Polar Spur. \\
12 & Synchrotron & $270^{\circ}-310^{\circ}$ & $+55^{\circ}-+70^{\circ}$ & Outermost section of North Polar Spur. \\
13 & Synchrotron & $350^{\circ}-5^{\circ}$ & $-35^{\circ}--50^{\circ}$ & Southern bulge in synchrotron sky. \\
14 & Synchrotron & $70^{\circ}-90^{\circ}$ & $+20^{\circ}-+30^{\circ}$ & A "weak" northern spur. \\
15 & Synchrotron & $76^{\circ}-84^{\circ}$ & $-30^{\circ}--50^{\circ}$ & A southern spur. \\
\hline
\end{tabular}

Table 1 Details of the 15 selected regions 

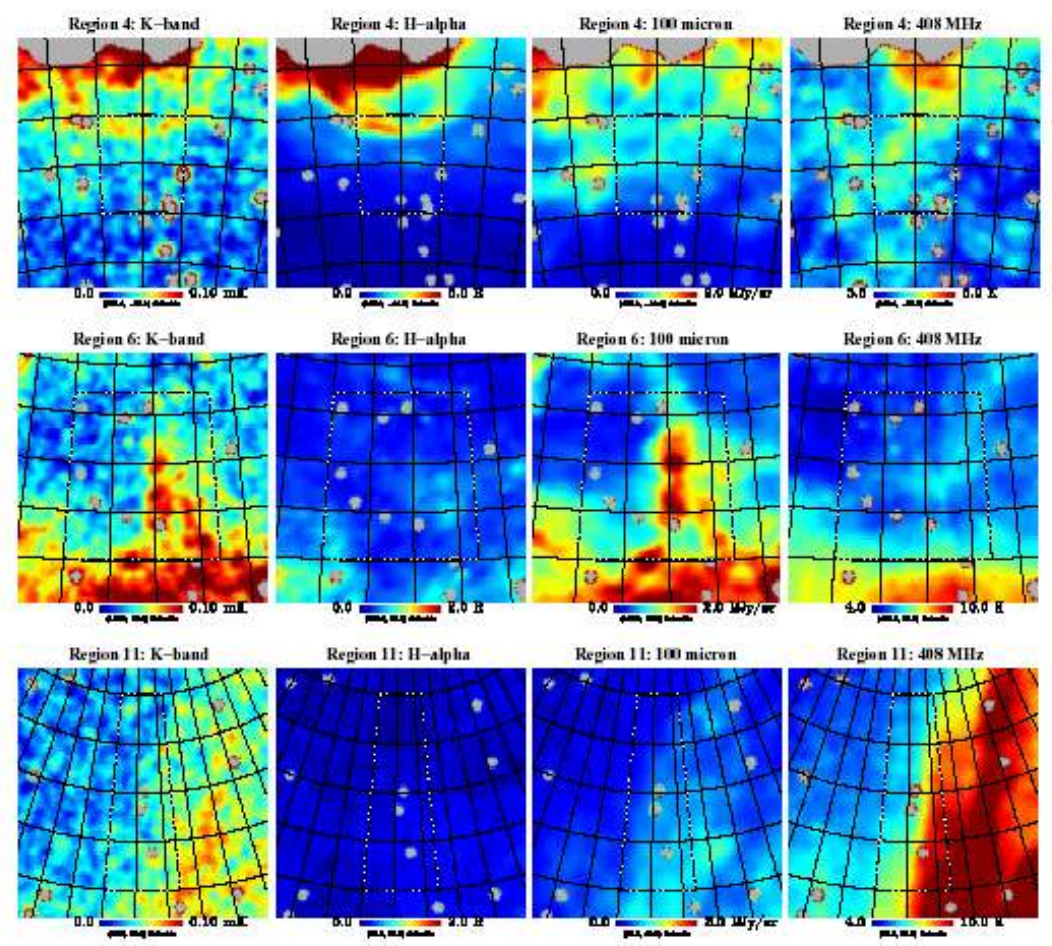

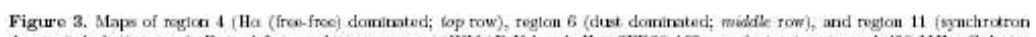

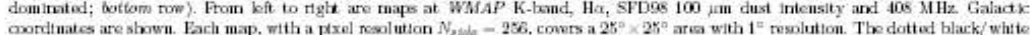

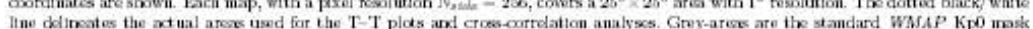
and extragatactte sources mask

Fig. 2 Maps of regions 4, 611 at K Band, H-alpha for free-free, 100 microns for dust and $408 \mathrm{MHz}$ for synchrotron.

\section{Analysis of the 15 regions}

Fig. 1 shows the position of the 15 selected regions overlaid on the Kp2 intensity mask and source mask (700 sources in total) used by the WMAP team (Bennett et al. 2003). Fig. 2 shows 3 regions (regions 4, 6 and 11) with an ILC-subtracted K-band, Ha, 100 micron and $408 \mathrm{MHz}$ data. The dominant foreground in each region (see Table 1) is clearly seen along with the correlated emission at K-band. The anomalous emission associated with dust is clearly detected in most of the 15 fields studied. Fields that are only weakly contaminated by synchrotron, free-free and CMB are studied; the anomalous emission correlated well with the Finkbeiner et al. (1999) model 8 predictions (FDS8) at $94 \mathrm{GHz}$, with an effective spectral index between 20 and $60 \mathrm{GHz}$, of beta $\sim-2.85$. Furthermore, the emissivity varies by a factor of $\sim 2$ from cloud to cloud.

CMB and Physics of the Early Universe - International Conference

Ischia, Italy

April 20-22, 2006 


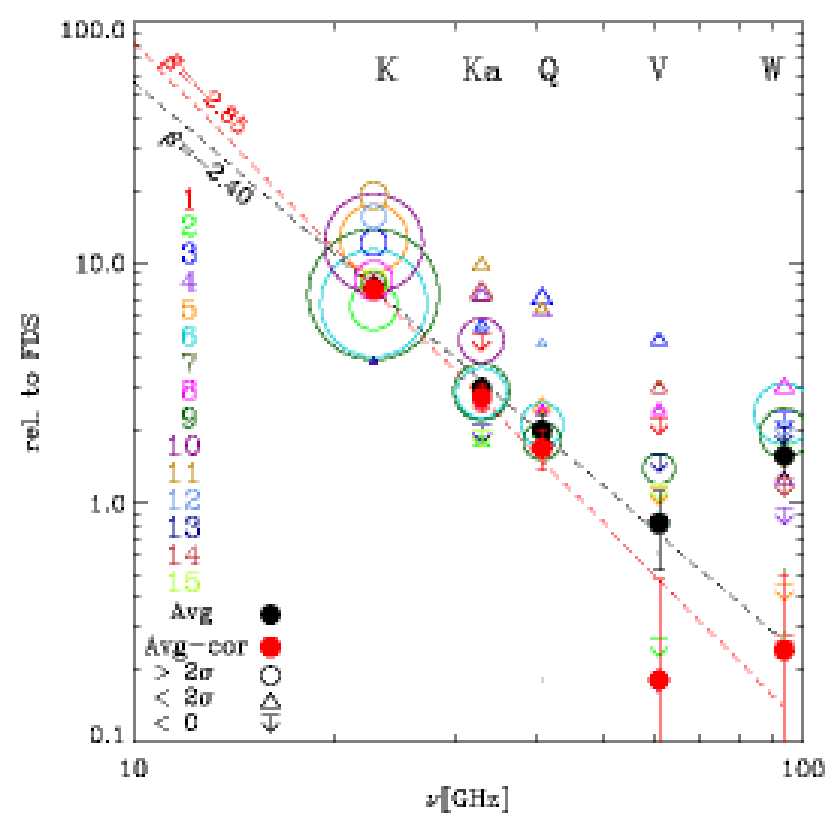

Fig. 3 Summary of dust emissivities (antenna temperature units relative to FDS8 at $94 \mathrm{GHz}$

Our analysis of the WMAP data indicates strongly that the dust-correlated emission at the low WMAP frequencies has a spectrum which is compatible with spinning dust; we find no evidence for a synchrotron component correlated with dust. The importance of these results for the correction of $\mathrm{CMB}$ data for Galactic foreground emission is discussed.

Further work is required to understand the origin of this variation in dust emissivity by using other physical properties of dust such as its size and temperature. New data in the critical radio frequency range $5-15 \mathrm{GHz}$ will be vital for a clearer definition of the anomalous dust spectrum. Polarisation data will be particularly important for understanding the physical mechanism that produces the anomalous emission which is expected to be polarized at different levels (e.g. Draine \& Lazarian 1999). For example, spinning dust emission is expected to be only weakly polarized, whereas the synchrotron emission is known to be highly polarised.

\section{References}

[1] Bennett C.L. et al., 2003, ApJS, 148, 97

[2] Davies R.D. et al., in press 2006

[3] Draine B.T., Lazarian A., 1999, ApJ, 512, 740

[4] Finkbeiner D.P., Davis, M., Schlegel D.J., 1999, ApJ, 524, 867 (FDS)

[5] Lazarian A., Draine B.T., 2000, ApJ, 536, L15 